\title{
Correction to: A randomized controlled trial of the impact of body-worn camera activation on the outcomes of individual incidents
}

\author{
Jessica Huff ${ }^{1} \cdot$ Charles M. Katz $^{1}$ - E. C. Hedberg ${ }^{2}$ \\ Published online: 17 August 2021 \\ (C) Springer Nature B.V. 2021
}

\section{Correction to: Journal of Experimental Criminology https://doi.org/10.1007/s11292-020-09448-5}

In the article "A randomized controlled trial of the impact of body-worn camera activation on the outcomes of individual incidents" by Jessica Huff, Charles M. Katz, and E. C. Hedberg, the Stata code and output referenced in Appendix A detailing the use of path models to estimate treatment effects were not included.

The online version of the original article can be found at https://doi.org/10.1007/s11292-020-09448-5

Jessica Huff

jlhuff2@asu.edu

1 Center for Violence Prevention and Community Safety, School of Criminology and Criminal Justice, Arizona State University, 411 N. Central Ave, Suite 680, Phoenix, AZ 85004-0685, USA

2 NORC at the University of Chicago, Boston, MA, USA 
- reg y z

\begin{tabular}{|c|c|c|c|c|c|c|}
\hline Source & SS & $d f$ & MS & Number of obs & $=$ & 20 \\
\hline Model & 76.05 & 1 & 76.05 & $\mathrm{Prob}>\mathrm{F}$ & $=$ & 0. 5394 \\
\hline Residual & 3496.9 & 18 & 194.272222 & R-squared & $=$ & 0.0213 \\
\hline Total & 3572.95 & 19 & 188.05 & $\begin{array}{l}\text { Aa] R-square } \\
\text { Root MSE }\end{array}$ & $\begin{array}{l}= \\
=\end{array}$ & $\begin{array}{r}-0.0331 \\
13.938\end{array}$ \\
\hline
\end{tabular}

\begin{tabular}{r|rrrrrr}
\hline$y$ & Coef. & Std. Err. & $t$ & $\mathrm{P}>|t|$ & [95\% Conf. Interval] \\
\hline z & -3.9 & 6.233333 & -0.63 & 0.539 & -16.99575 & 9.195747 \\
_cons & 57 & 4.407632 & 12.93 & 0.000 & 47.73991 & 66.26009 \\
\hline
\end{tabular}

The regression Stata code and output referenced in the appendix are shown here:The instrumental variable regression code and results referenced within the appendix are shown here:

- ivregress 2 sls y $(x=z)$

Instrumental variables (2SLS) regression

$\begin{array}{llr}\text { Number of obs } & = & 20 \\ \text { Wald chi2(1) } & = & 0.41 \\ \text { Prob > chi2 } & = & 0.5201 \\ \text { R-squared } & = & . \\ \text { Root MSE } & = & 13.557\end{array}$

\begin{tabular}{r|rrcrrr}
\hline$y$ & Coef. & Std. Err. & $z$ & $P>|z|$ & [95\% Conf. Interval] \\
\hline$x$ & -5.571429 & 8.661317 & -0.64 & 0.520 & -22.5473 & 11.40444 \\
_cons & 58.11429 & 5.646488 & 10.29 & 0.000 & 47.04737 & 69.1812 \\
\hline
\end{tabular}

Instrumented: $\mathrm{x}$

Instruments: $\quad \mathrm{z}$ 
Finally, the sem code and results referenced within the appendix are presented here:

- $\operatorname{sem}(x->y),(z->x),, \operatorname{cov}\left(e \cdot x^{*} e \cdot y\right)$ nocapslatent

Endogenous variables

Observed: $\mathrm{x}$ y

Exogenous variables

Observed: $\quad$ z

Fitting target model:

Iteration 0: $\quad \log$ likelihood $=-102.02278$

Iteration 1: $\quad \log$ likelihood $=-102.02278$

Structural equation model

Number of obs =

Estimation method $=\mathrm{ml}$

Log likelihood $\quad=-102.02278$

\begin{tabular}{|c|c|c|c|c|c|c|}
\hline & Coef. & $\begin{array}{c}\text { OIM } \\
\text { Std. Err. }\end{array}$ & z & $P>|z|$ & [95\% Conf. & Interval] \\
\hline \multicolumn{7}{|l|}{$\begin{array}{l}\text { Structural } \\
\mathrm{x}\end{array}$} \\
\hline$z$ & .7 & .1581139 & 4.43 & 0.000 & .3901025 & 1.009898 \\
\hline _cons & .2 & .1118034 & 1.79 & 0.074 & -.0191306 & .4191306 \\
\hline \multicolumn{7}{|l|}{$y$} \\
\hline$x$ & -5.571429 & 8.661317 & -0.64 & 0.520 & -22.5473 & 11.40444 \\
\hline _cons & 58.11429 & 5.646488 & 10.29 & 0.000 & 47.04737 & 69.1812 \\
\hline $\operatorname{var}(e \cdot x)$ & .125 & .0395285 & & & .0672568 & .2323184 \\
\hline $\operatorname{var}(e \cdot y)$ & 183.7951 & 61.44834 & & & 95.44456 & 353.9294 \\
\hline $\operatorname{cov}(e \cdot x, e \cdot y)$ & 1.151429 & 1.545047 & 0.75 & 0.456 & -1.876809 & 4.179666 \\
\hline
\end{tabular}

LR test of model vs. saturated: $\operatorname{chi2}(\theta)=$

0.00 , Prob > chi2 $=$

Publisher's note Springer Nature remains neutral with regard to jurisdictional claims in published maps and institutional affiliations. 